\title{
ENDODONTIC RETREATMENT OF DENTES GEMINATI -
}

\section{A CASE REPORT}

\author{
Polina Velcheva ${ }^{1}$, Metodi Abadzhiev², Plamen Nenkov ${ }^{3}$ \\ ${ }^{1}$ Department of Conservative Dentistry and Oral Pathology, Faculty of Dental Medicine, \\ Medical University of Varna \\ ${ }^{2}$ Department of Prosthetic Dentistry and Orthodontics, Faculty of Dental Medicine, \\ Medical University of Varna \\ ${ }^{3}$ Department of Periodontology and Implant Dentistry, Medical University of Varna
}

\begin{abstract}
INTRODUCTION: The shape of deciduous and permanent teeth can differ morphologically from that which is considered normal, partially or entirely. The variations in tooth shape can be hereditary or caused by a disease or trauma. Gemination, for example, means that two separate morphological units were created by division of the tooth germ. This is an extremely rare condition with a prevalence of $0.2-0.5 \%$.
\end{abstract}

AIM: A patient with symptomatic apical periodontitis of a previous endodontically treated tooth 21 was complaining of swelling and pain in the frontal area of maxilla. The basic features and specific appearance of geminated teeth were present - an enlarged mesio-distal diameter of the tooth crown, a groove on the incisal edge, a shallow or deeper groove on the vestibular and oral surface, which divides the tooth into two, usually unequal parts.

MATERIALS AND METHODS: Isolation of the tooth was made by OpraDam (Ivoclar Vivadent). For the mechanical debridement of this complex root canal system, we used H-files and rotary files Pro Taper Next till X3 file. Cleaning was done by passive ultrasonic activation and photon-induced photoacoustic streaming by Er-YAG laser of $5.25 \% \mathrm{NaOCl}, 16 \%$ EDTA and $2 \% \mathrm{XX}$. After double application of $\mathrm{Ca}(\mathrm{OH})_{2}$ as an intracanal medicament for at least 1 week, the sinus track and symptoms were gone. The obturation technique was warm vertical condensation with System B and Obtura (SybronEndo).

CONCLUSION: Dentes geminati are extremely rare and difficult to treat endodontically but after proper cleaning, shaping and obturation of this complex root canal system with 2 main root canals with huge isthmus between them and lateral canals we can expect healing.

Keywords: endodontic treatment, dens geminate, Er-YAG laser, warm vertical compaction

Address for correspondence:

Polina Velcheva

Faculty of Dental Medicine

Medical University of Varna

84 Tsar Osvoboditel Blvd.

9000 Varna

e-mail:polina_velcheva87@abv.bg

Received: April 5, 2017

Accepted: January 19, 2017

\section{INTRODUCTION}

Double teeth or twinning teeth are morphological dental anomalies, characterized by formation of clinically wide teeth. Gemination teeth, the Latin name of these types of teeth, are associated with clinical problems such as poor esthetics, spacing problems and caries susceptibility. Management of such cases requires a comprehensive knowledge of the clinical entity as well as the problems associat- 
ed with it. This report presents a case of a permanent maxillary incisor 21 with symptomatic apical periodontitis after improper endodontic treatment because of a complex root canal system. The treatment plan included additional methods for proper disinfection and cleaning of the root canal space like passive ultrasonic activation and photon-induced photoacoustic streaming (PIPS) of irrigation solutions. After correct medication and 3D obturation of the root canal system, we provided environment for complete healing of the sinus track and periapical lesion.

The form of primary and permanent teeth can differ morphologically from that what is considered normal, completely or partially. The changes in tooth from can be hereditary or caused by a disease or trauma. Examples for "double teeth" are gemination and fusion and the importance from a practical point of view is for a differential diagnosis to be made. Gemination means that two separate morphological units were created by division of the tooth germ. Fusion is a union of one or more teeth during development. The results of a study in Zagreb have shown that double teeth are extremely rare with a prevalence of $0.2 \%$ and $57.2 \%$ of them were fused and $42.9 \%$ - geminated (1).

Twin teeth - dentes geminati, can be placed into the group of twinning. There is a difference between the notion of twinning and that of supernumerary teeth. Twinning develops from a tooth germ within one tooth follicle, while supernumerary teeth form from an overproliferation of cells at certain locations in the developing tooth band (dental lamina). If one tooth germ forms two equal teeth by division schizodontia, we speak of germination. Gemination is more frequent in primary than in permanent teeth. Teeth with irregularities, such as gemination, have a characteristic appearance - the mesio-distal diameter of the clinical crown is larger than normal, and from the incisal edge to the apex of the root a grove of unequal depth divides the tooth into two, usually unequal parts (2). Fusion is quite the opposite process - synodontia, which is uniting two or more tooth germs (separate tooth organs during embryonic development). Fusing can be complete (total) or incomplete (partial), it depends on the time when the force causing the narrowing of the space between the tooth germs was at work. When the fusion is complete one tooth is formed and this leads to a reduced number in the dental arch. It is quite difficult to recognize the kind of abnormality. It is based on clinical and radiographic evaluation. The clinical appearance of a crown of fused teeth can sometimes be identical to the finding of geminated teeth. Milazzo and Alexander (3) have, for the purpose of a differential diagnosis, suggested counting of teeth: a normal number of teeth in the dental arch indicates gemination, a reduced number - fusion. An exception are cases where there is a normal tooth fused with a supernumerary one, or when there is a gemination in a dental arch with hypodontia (4). The etiology of twin teeth formation is generally unclear. Many theories connect environmental influences, genetics, trauma, systemic disease, the lack of vitamins and lack of space in the dental arch as possible causes of this anomaly.

\section{MATERIALS AND METHODS}

In this particular case there was a patient with symptomatic apical periodontitis of previous endodontically treated tooth 21 . The patient was 17 -yearold boy with complaints of swelling and pain in frontal area of maxilla. The basic features and specific appearance of geminated teeth were present in both of the maxillary central incisors - an enlarged mesiodistal diameter of the tooth crown, a groove on the incisal edge, a shallow or deeper groove on the vestibular and oral surface, which divides the tooth into two, usually unequal, parts.

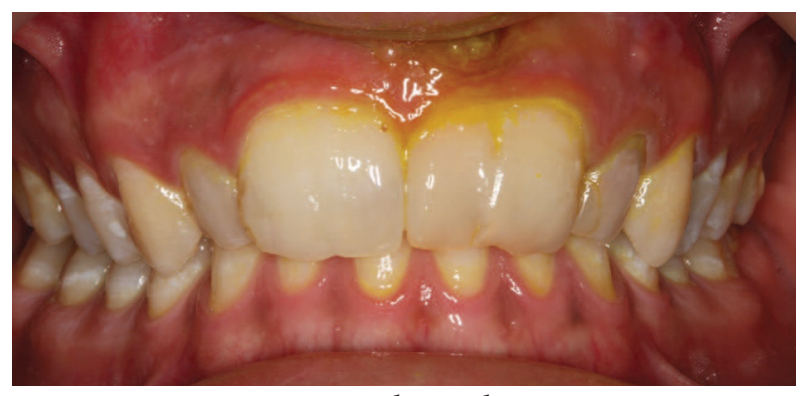

Fig. 1. Intraoral initial situation

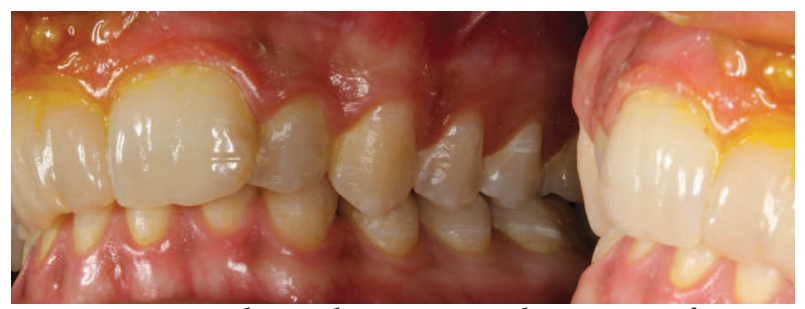

Fig. 2. Intraoral initial situation with presence of sinus track 
Polina Velcheva, Metodi Abadzhiev, Plamen Nenkov

There were radiographic and clinical sings of failed endodontic treatment because of a complex root canal system, which was inappropriately cleaned and disinfected. The diagnosis of tooth 21 was symptomatic apical periodontitis with active sinus track.

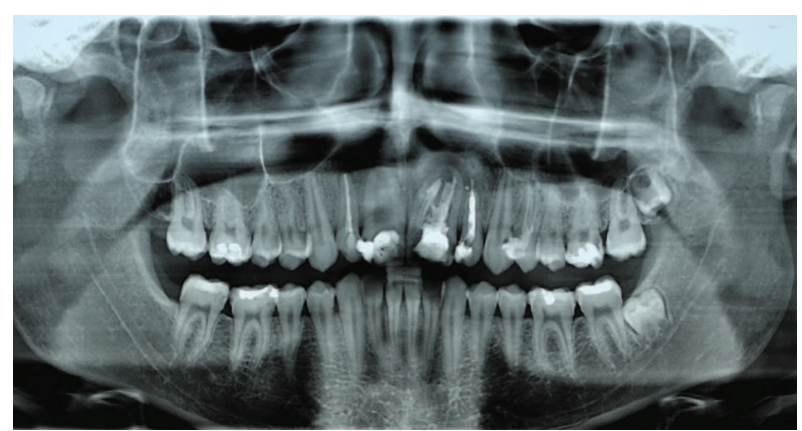

Fig. 3. Pre-op X-ray with large periapical radiolucency and incorrect filling of complex root canal space

After proper isolation of the tooth by OpraDam (Ivoclar Vivadent ${ }^{\natural}$ ) without clamps, two orifices with large isthmus between them were found.

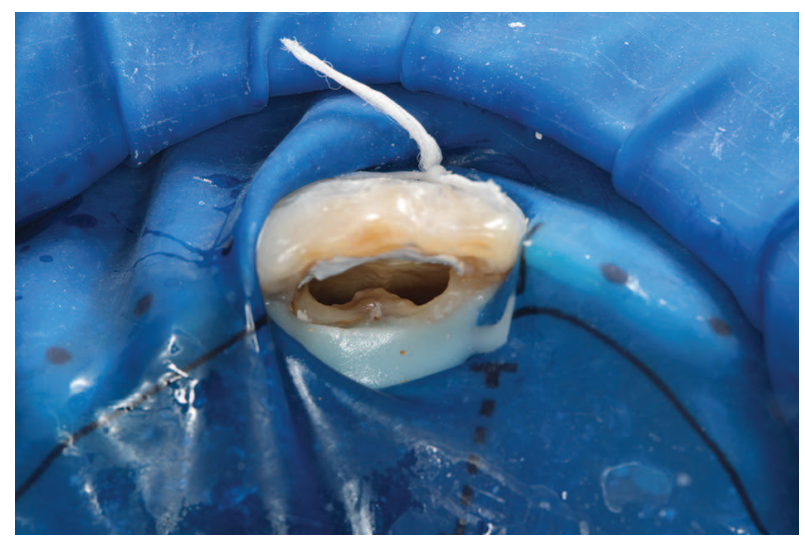

Fig. 4. Intraoral view of orifices on tooth 21

The goal of the endodontic treatment is to obtain effective cleaning and decontamination of the smear layer, bacteria and their bio-products within the root canal system. Clinically, traditional endodontic techniques use mechanical instruments as well as ultrasonic and chemical irrigation in an attempt to shape, clean and completely decontaminate the endodontic system, but still fall short of successful removal of all of the infective microorganisms and debris. This is because of the complex root canal anatomy and the inability of common irrigants to penetrate into the lateral canal and the apical ramifications. It seems, therefore, appropriate to use new materials, techniques and technologies that can improve the cleaning and decontamination of these anatomical areas.

For the mechanical debridement of this complex root canal system, we used manual $\mathrm{H}$-files only to remove old gutta-percha points and rotary files Pro Taper next till X3. There were many anastomoses and connections along the main canal which are impossible for the endodontic instruments to reach. This is why we decided to clean the root canal space by passive ultrasonic activation and photon-induced photoacoustic streaming (PIPS) by Er-YAG laser of irrigation solutions $-5.25 \% \mathrm{NaOCl}, 16 \%$ EDTA and 2\% XX.

The disinfecting action of PIPS is very effective both on the root surface, lateral canals and the dentinal tubules, as confirmed by bacterial studies as well as SEM and confocal studies (5-7).

Clinical trials showed that the PIPS technique greatly simplifies root canal therapy while facilitating the search for the apical terminus, debriding and maintaining patency. The ability, of PIPS to three-dimensionally debride and decontaminate dental tubules presents the clinician with the possibility to effectively deliver treatments in less time and with less need to enlarge the canal system, allowing for a more minimally invasive and biomimetic preparation which can then be obturated three dimensionally $(5,8,9)$.

On the first appointment, gutta-percha points from the previous endodontic treatment were successfully removed. Disinfection was made by passive ultrasonic activation of irrigation solutions in the following sequence $-5.25 \% \mathrm{NaOCl}$ activated for $20 \mathrm{sec}-$ onds, saline, solution of $16 \%$ EDTA activated for 20 seconds, saline, double irrigation with $5.25 \% \mathrm{NaOCl}$ also activated for 20 seconds, again saline and solution of 16\% EDTA activated for 20 seconds then saline and for a final rinse $2 \%$ solution of chlorhexidine was used. The canals were left empty for 24 hours and during the next two visits the same irrigation protocol was followed using calcium hydroxide paste as intracanal medication.

After double application of $\mathrm{Ca}(\mathrm{OH})_{2}$ as intracanal medicament for at least 1 week the sinus track and symptoms were gone.

During the fourth appointment, the same irrigation protocol was used with photon-induced pho- 


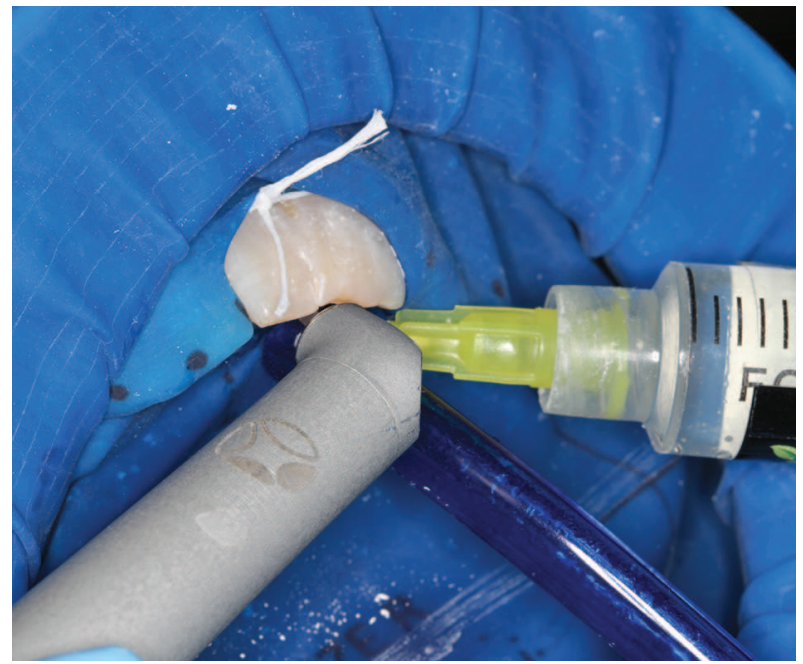

Fig. 5. Activating of irrigation solutions with photon-induced photoacoustic streaming (PIPS) by Er-YAG laser

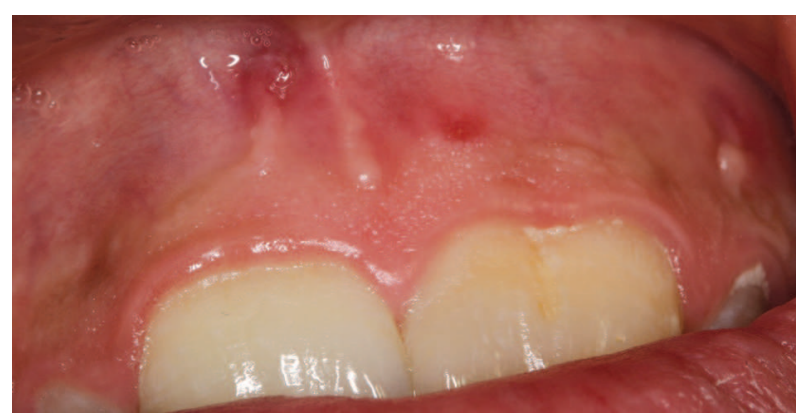

Fig. 6. Healing of sinus track after intracanal dressing of $\mathrm{Ca}(\mathrm{OH})_{2}$

toacoustic streaming (PIPS) by Er-YAG laser. For the final rinse $95 \%$ ethanol was used and the canal was wiped dry with paper points 35/06. The obturation technique was warm vertical condensation with $\mathrm{AH}$ plus sealer, System B and Obtura (SybronEndo).

\section{RESULTS}

In the post-op X-ray it can be seen that except for two main canals there is an apical delta and lateral canal in the middle third. The patient is asymptomatic and after appropriate debridement and obturation of the root canal space we provided an excellent environment for the regeneration in the periapical area of this tooth with congenitally formed anomaly.

\section{DISCUSSION}

The alternative of the conservative treatment was extraction, orthodontic treatment to increase the M-D space between teeth 12 and 22, bone augmen-

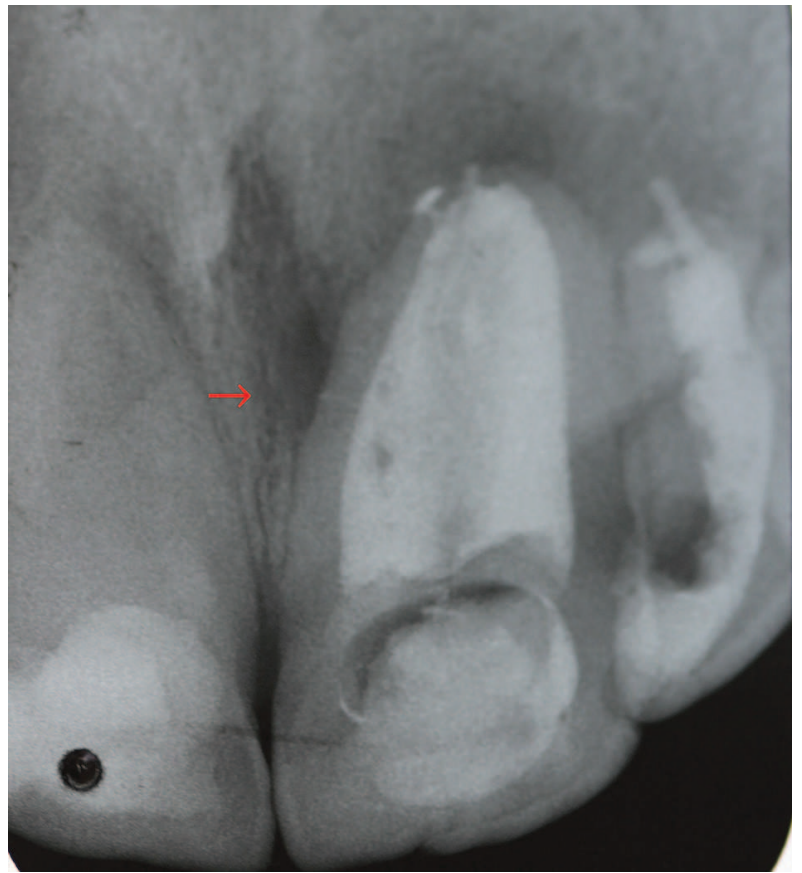

Fig. 7. Post-op X-ray with presence of lateral canal and apical delta

tation and implant placement. This treatment plan is probably more esthetic but too invasive and with a long duration (10).

Clinically, the abnormalities of double teeth manifest themselves in higher caries incidence, malocclusion, changes in the length of the dental arch, periodontal diseases, hyper/hypodontia of the successional tooth, anomalies in the eruption of the successional tooth and poor esthetics.

If double teeth are diagnosed earlier in childhood prophylactic precaution measures should be taken in order to avoid complications. When there are indications for endodontic treatment, additional methods for root canal disinfection should be used and it is recommended for the patient to be treated by an endodontist.

\section{CONCLUSION}

Dentes geminati are extremely rare and difficult to treat endodontically but after proper cleaning, shaping and obturation of this complex root canal system with 2 main root canals with huge isthmus between them and lateral canals we can expect complete healing. 


\section{REFERENCES}

1. Knezevic A, Travan S, Tarle Z, Sutalo J, Janković B, Ciglar I. Double Tooth. Coll Antropol. 2002;26(2):667-72.

2. Chipashvili N, Vadachkoria D, Beshkenadze E. Gemination or fusion? -challenge for dental practitioners (case study). Georgian Med News. 2011;5(194):28-33.

3. Milazzo A, Alexander SA. Fusion, gemination, oligodontia, and taurodontism.J Pedod. 1982; 6(2):194-9.

4. Kulkarni VK, Ragavendra TR, Deshmukh J, Vanka A, Duddu MK, Patil AKG. Endodontic treatment and esthetic management of a primary double tooth with direct composite using silicone buildup guide. Contemp Clin Dent. 2012; 3(Suppl1): 92-5. doi: 10.4103/0976-237X.95114

5. Olivi G, DiVito E. REVIEW Photoacoustic Endodontics using PIPS $^{\mathrm{ms}}$ : experimental background and clinical protocol. Journal of the Laser and Health Academy. 2012;1:22-5.

6. DiVito E, Lloyd A. ER:YAG laser for 3-dimensional debridement of canal systems: use of photon-induced photoacoustic streaming. Dent Today.2012; 31(11):122, 124-7.

7. Arslan H, Capar ID, Saygili G, Gok T, Akcay M. Effect of photon-initiated photoacoustic streaming on removal of apically placed dentinal debris. Int Endod J. 2014; 47(11): 1072-7. doi: 10.1111/iej.12251

8. Arslan H, Akcay M,Capar ID. An in vitro comparison of irrigation using photon-initiated photoacoustic streaming, ultrasonic, sonic and needle techniques in removing calcium hydroxide. Int Endod J. 2015; 48(3):246-51. doi: 10.1111/iej.12306

9. Lloyd A, Uhles JP, Clement DJ, Garcia-Godoy F. Elimination of intracanal tissue and debris through a novel laser-activated system assessed using highresolution micro-computed tomography: a pilot study. J Endod. 2014; 40(4): 584-7. doi: 10.1016/j. joen.2013.10.040

10. Ghijselings E, Van Gastel J, Verdonck A, Carels C. Clinical problems with a double tooth and a macrodont in the maxillary anterior area: a case report. Aust Orthod J.2014; 30(2):221-9. 